\title{
ESTIMATION OF GROWTH PARAMETERS OF FIVE FISH SPECIES (ACTINOPTERYGII) CAUGHT IN THE CENTRAL AMAZON
}

\author{
Ana GUERREIRO ${ }^{1 *}$, Sidineia AMADIO ${ }^{2}$, Nídia FABRÉ ${ }^{3}$, and Vandick BATISTA ${ }^{3}$ \\ ${ }^{1}$ Pós-graduação em Biologia de Água Doce e Pesca Interior, Instituto Nacional de Pesquisas da Amazônia, \\ Petrópolis, Manaus, Amazonas, Brazil \\ ${ }^{2}$ Coordenação de Biodiversidade, Instituto Nacional de Pesquisas da Amazônia, Petrópolis, Manaus, \\ Amazonas, Brazil \\ ${ }^{3}$ Instituto de Ciências Biológicas e da Saúde, Universidade Federal de Alagoas, Campus A. C. Simões, Tabuleiro dos \\ Martins, Maceió, Alagoas, Brazil
}

Guerreiro A., Amadio S., Fabré N., Batista V. 2018. Estimation of growth parameters of five fish species (Actinopterygii) caught in the central Amazon. Acta Ichthyol. Piscat. 48 (3): 303-313.

\begin{abstract}
For some tropical fishes, the information on growth parameters is still scarce, and few or no records are available in FishBase. Therefore, the objective of this study was to estimate the growth curves for Brycon amazonicus (Spix et Agassiz, 1829), Piaractus brachypomus (Cuvier, 1818), Prochilodus nigricans Spix et Agassiz, 1829, Semaprochilodus insignis (Jardine, 1841), and Semaprochilodus taeniurus (Valenciennes, 1821), and to build the auximetric plots for each of the families to which these species belong: Characidae, Prochilodontidae, and Serrasalmidae. Samples were obtained from commercial catches landed in the Port of Manaus. Growth parameters were estimated using the Electronic Length Frequency Analysis (ELEFAN) routine of the Length Frequency Distribution Analysis (LFDA) program. Twenty-six sets of growth parameters were thus estimated, and 66 further sets were located in the literature and FishBase. Prochilodontidae and Serrasalmidae showed a strong inverse relation between the variables composing the auximetric plots.
\end{abstract}

Keywords: tropical fishes, commercial fishery, length frequency data, growth coefficient, asymptotic length

\section{INTRODUCTION}

Growth is one of the most critical measurable characteristics of individuals, stocks, and species, and it is fundamental to our understanding of the life histories, demographics, ecosystem dynamics, and sustainability of fisheries (Pardo et al. 2013). Fishes are the primary food source of the Amazon's local inhabitants, with some areas showing the highest consumption rates in the world (with fish consumed six out of every seven days, at a mean rate of $169 \mathrm{~kg}$ per person per year) (Isaac et al. 2015). The dynamics of fish stocks may alter due to environmental changes (Barletta et al. 2010), overfishing, climate change, pollution, deforestation, etc. (Li et al. 2011, Freitas et al. 2013). However, growth parameters of fishes from the central Amazon-key indicators that will enable the assessment of the impact of such processes on fish populations - are still scarce for Brycon amazonicus (Spix et Agassiz, 1829), Piaractus brachypomus (Cuvier, 1818), Prochilodus nigricans Spix et Agassiz, 1829, Semaprochilodus insignis (Jardine, 1841), and Semaprochilodus taeniurus (Valenciennes, 1821). This study aimed to estimate the von Bertalanffy growth parameters for five fish species of significant commercial interest within six rivers in the Amazon region. In addition, the study built auximetric plots of each of the three families to which these species belong (Characidae, Prochilodontidae, and Serrasalmidae).

\section{MATERIAL AND METHODS}

The samples were obtained from the catches of commercial fisheries along the Amazon, Japurá, Juruá, Madeira, Negro, and Purus rivers that landed at the Port of Manaus, Brazil $\left(03^{\circ} 08^{\prime} 47^{\prime \prime} \mathrm{S}, 60^{\circ} 06^{\prime} 35^{\prime \prime} \mathrm{W}\right)$. The sampling protocol was designed to measure the fish length of a total of 300 individuals per month. Thus, 30 individuals per species per night had their fork length (FL, cm) measured (as the caudal fin was often damaged) on 10 randomly selected days within a month-long period. To ensure a 'knife-edge' selection, only those fish captured with 20 $\mathrm{mm}$ mesh seine net were used in this analysis (see Batista and Freitas (2003) for technological fishing details). Five out of the 10 species with the highest catches in the 
database were examined, namely Prochilodus nigricans, Semaprochilodus insignis, Semaprochilodus taeniurus, Brycon amazonicus, and Piaractus brachypomus.

Growth parameters were estimated using the seasonal growth curve proposed by Hoenig and Hanumara (1990). The equation is:

$$
L(t)=L \infty\left\{1-e^{-\left[K(t-t 0)+\frac{K C}{2 \pi} \operatorname{SIN} 2 \pi(t-t s)-\frac{K C}{2 \pi} \operatorname{SIN} 2 \pi(t 0-t s)\right]}\right\}
$$

where $K$ is the growth coefficient, $L_{\infty}$ the asymptotic size, $t_{0}$ the theoretical age at zero length, $C$ the relative amplitude of seasonal oscillations, and the $t_{\mathrm{s}}$ describes the phase of seasonal oscillations.

Finally, the Winter Point (WP) (García-Berthou et al. 2012) was determined using the following equation:

$$
\mathrm{WP}=t \mathrm{~s}+0.5
$$

The ELEFAN routine of the LFDA program (Kirkwood et al. 2001) was used with sets of between two and six years, depending on data availability. A growth curve was fitted using any arbitrary 'seed' input values of $L_{\infty}$ and $K$ (Pauly and David 1981).

\section{RESULTS}

In some years, only juveniles were caught. Thus, the length at first maturity available in the literature, as well as the maximum length found here, were used to ensure that the data used - and, consequently, the estimates foundwere reliable. Thus, we only used years in which data were available for both juveniles and adults. Moreover, we used the age at first maturity available in the literature to evaluate the reliability of the growth curve estimates for each species (Table 1).

Finally, as Pauly (1998) reported an inverse pattern between the asymptotic lengths and the growth coefficients of tropical fish, auximetric plots were built for each of the three families to which the species studied here belong: Characidae, Prochilodontidae, and Serrasalmidae. In addition, we utilised other growth parameters found in FishBase (Froese and Pauly 2018), and 21 other studies (Ruffino and Isaac 1995, Isaac and Ruffino 1996, Lizama and Ambrósio 2003, Penna et al. 2005, Silva and Stewart 2006, Cunha et al. 2007, Gomiero et al. 2007, Peixer et al. 2007, Santos Filho and Batista 2009, Carmassi et al. 2011, Bevilaqua and Soares 2010, Isaac et al. 2012, Lourenço et al. 2012, Tondato et al. 2012, Vicentin et al. 2012, Pérez-Lozano and Aniello 2013, Sousa et al. 2013, Ambrósio et al. 2014, Catarino et al. 2014, Vaz unpublished", Villacorta-Corrêa unpublished ${ }^{* *}$ ) (Table 2), for these same families. The correlation between the two variables of each of the auximetric plots $\left(\log _{10}\right.$ asymptotic length and $\log _{10}$ growth coefficient) was tested using the non-parametric Spearman rs test.

The growth parameter estimates are shown in Table 3, and the LFDA growth curves in Figs. 1-7. Additional information used in the estimation of these parameters is available in Table 1.

\section{DISCUSSION}

Fish exhibit differences in growth as a result of being subjected to environmental fluctuations (King 2007). Amazonian fish are also subject to the annual flood regime, as well as periodical natural climatic phenomena (Junk 1983, Tomasella et al. 2012, Camacho Guerreiro et al. 2016, Guerreiro 2017). The variations observed in the growth parameters of some of the species sampled appear to accord with the high degree of environmental variation observed in this type of ecosystem.

No trend was observed in the auximetric plot of Characidae (Spearman rs test, $r_{\mathrm{s}}=-0.14, P=0.60$ ) (Fig. 8A). However, for the Prochilodontidae (Spearman rs test, $r_{\mathrm{s}}=-0.67, P<0.001$ ) (Fig. $8 \mathrm{~B}$ ) and Serrasalmidae (Spearman rs test, $r_{\mathrm{s}}=-0.65, P<0.001$ ) (Fig. $8 \mathrm{C}$ ) families, a significant and inverse relation was found between the growth parameters. Growth parameters were found in the literature for only 10 species out of the 1113 species belonging to the Characidae (Froese and Pauly 2018). Thus, great effort is required to fill this research gap before it will be possible to build an auximetric plot for this family.

Finally, the pattern observed here between the growth parameters of Prochilodontidae and Serrasalmidae was similar to that recorded by Pauly (1998), supporting the reliability of the estimates obtained. In fact, the clusters of points on the auximetric plot seem to reflect similarities between the various species within these families.

\section{ACKNOWLEDGEMENTS}

We thank all fishers that provided information about catches and the team that collected this information; Elsa Guerreiro for reviewing the English of this paper; and Victor Silva for editing figures in Adobe Illustrator. This study was funded by Coordination for the Improvement of Higher Education Personnel (CAPES) (AICG grant), the Brazilian Innovation Agency (FINEP), and the [Brazilian] Ministry of Fisheries and Aquaculture (MPA) (grant No. 01.10.0770.00).

\section{REFERENCES}

Ambrósio A.M., Balbi T.J., Francisco T.M., Gomes L.C., Zuliani M.S., Okada E.K. 2014. Aging and growth parameter from the Piaractus mesopotamicus (pacu) from the Cuiabá River, Mato Grosso, Brazil.

\footnotetext{
*Vaz M. 2001. Problemas no ajuste da curva de crescimento do pacu, Piaractus mesopotamicus (Holmberg, 1887) (Pisces: Characidae), e seu manejo no Pantanal Mato-Grossense. [Problems in growth curve fitting of Pacu, Piaractus mesopotamicus (Holmberg, 1887) (Pisces: Characidae), and its management in Pantanal of Mato Grosso.] Doctoral thesis. São Paulo State University, Jaboticabal, Brazil. [In Portuguese.]

${ }^{* *}$ Villacorta-Corrêa M.A. 1997. Estudo de idade e crescimento do tambaqui Colossoma macropomum (Characidae) no Amazonas Central, pela análise de marcas sazonais nas estruturas mineralizadas e microestruturas nos otólitos. [Study of age and growth of Colossoma macropomum (Characiforms: Characidae) in Central Amazonas, through the seasonal marks analyses observed in the mineralized structure and microstructure of otoliths.] Doctoral thesis. INPA, Manaus, Brazil. [In Portuguese.]
} 
A

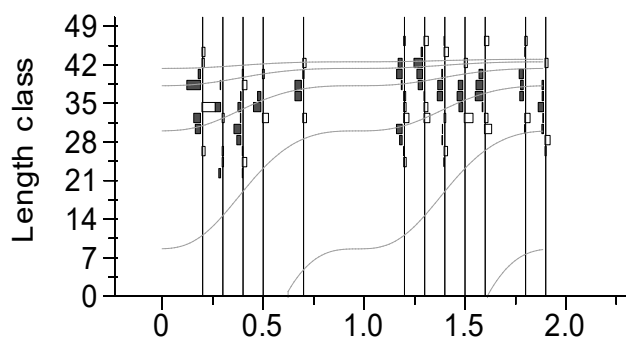

C

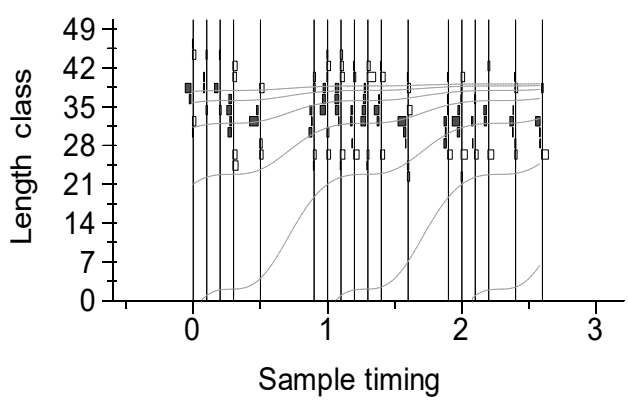

B

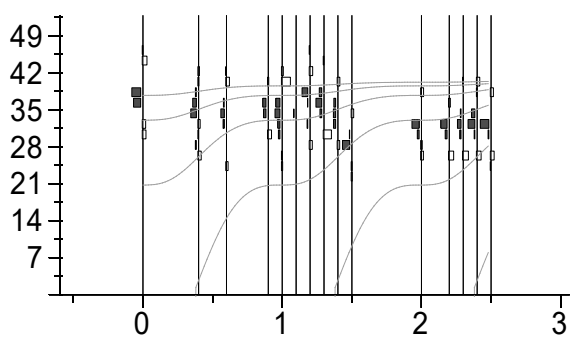

D

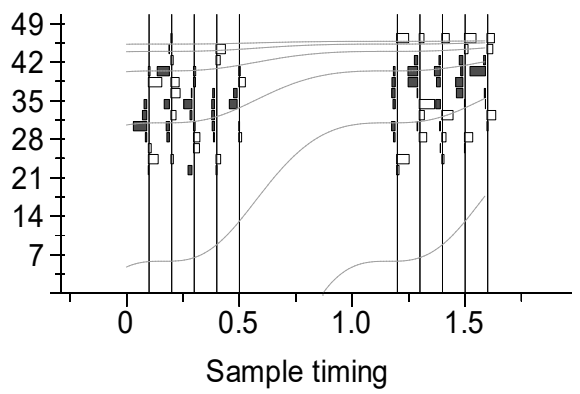

Fig. 1. Growth curves of Brycon amazonicus caught in three Amazonian rivers: (A) Amazonas 2003-2004, (B) Japurá 1998-2000, (C) Juruá 1998-2000, and (D) Purus 2003-2004

A

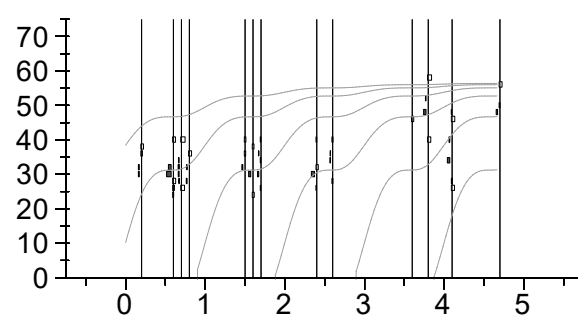

$\mathbf{B}$
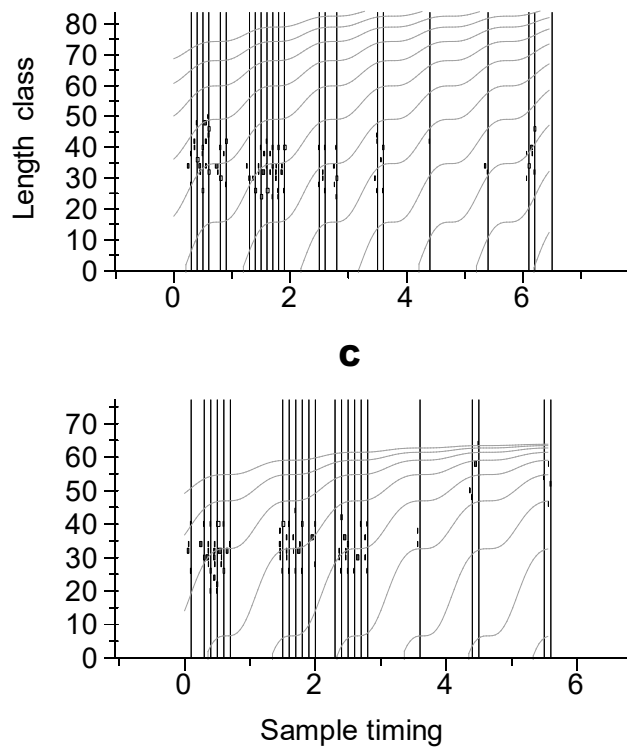

Fig. 2. Growth curves of Piaractus brachypomus caught in three Amazonian rivers: (A) Amazonas 2000-2004, (B) Japurá 1998-2004, and (C) Purus 1999-2004
Iheringia. Série Zoologia 104 (4): 413-417. DOI: 10.1590/1678-476620141044413417

Barletta M., Jaureguizar A.J., Baigun C., Fontoura N.F., Agostinho A.A., Almeida-Val V.M.F., Val A.L., Torres R.A., Jimenes-Segura L.F., Giarrizo T., Fabré N.N., Batista V.S., Lasso C., Taphorn D.C., Costa M.F., Chaves P.T., Vieira J.P., Corrêa M. 2010. Fish and aquatic habitat conservation in South America: A continental overview with emphasis on neotropical systems. Journal of Fish Biology 76 (9): 2118-2176. DOI: 10.1111/j.1095-8649.2010.02684.x

Batista V.S., Freitas V.S. 2003. O descarte de pescado na pesca com rede de cerco no baixo rio Solimões, Amazônia central. [Discards of fish in the purse-seine fishery in the lower Solimões River, central Amazon.] Acta Amazonica 33 (1): 127-143. [In Portuguese.]

Bevilaqua D.R., Soares M.G.M. 2010. Crescimento e mortalidade de Pygocentrus nattereri Kner, 1985 em lagos de várzea da região de Manacapuru, Amazônia. [Growth and mortality of Pygocentrus nattereri Kner, 1985 along the floodplains of Manacapuru, Amazonia.] Revista Brasileira de Engenharia de Pesca 5 (2): 43-52. [In Portuguese.]

Camacho Guerreiro A.I., Ladle R.J., Batista V.S. 2016. Riverine fishers' knowledge of extreme climatic events in the Brazilian Amazonia. Journal of Ethnobiology and Ethnomedicine 12 (1): e50. DOI: 10.1186/s13002016-0123-x

Carmassi A.L. Rondineli G.R., Gomiero L.M., Braga F.M.S. 2011. Crescimento e mortalidade de Oligosarcus hepsetus (Cuvier, 1829) (Characiformes, Characidae) no Parque Estadual da Serra do Mar, Núcleo Santa Virgínia, São Paulo, Brasil. [Growth 
and mortality of Oligosarcus herpsetus (Cuvier, 1829) (Characiforms, Characidae) in Serra do Mar State Park, Santa Virginia Unit, São Paulo, Brazil.] Biota Neotropica 11 (2): 40-43. [In Portuguese.] DOI: 10.1590/S1676-06032011000200004

A

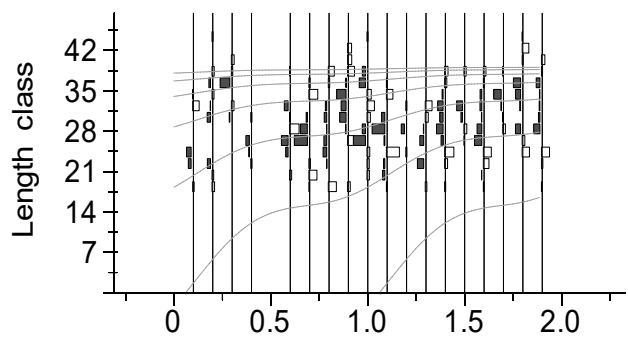

C

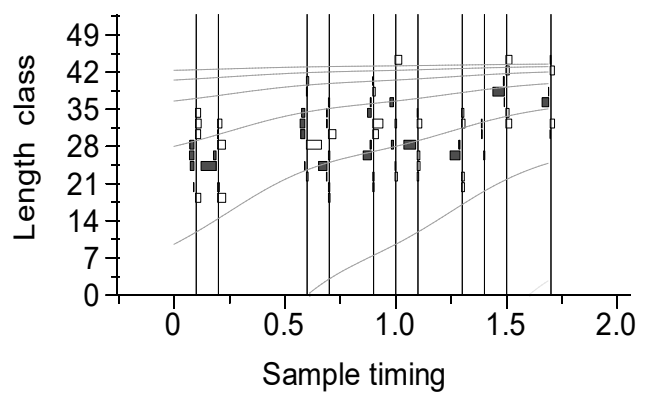

Catarino M.F., Campos C.P., Souza R.G.C., Freitas C.E.C. 2014. Population dynamics of Prochilodus nigricans caught in Manacapuru Lake (Amazon basin, Brazil). Boletim do Instituto de Pesca 40 (4): 589-595.
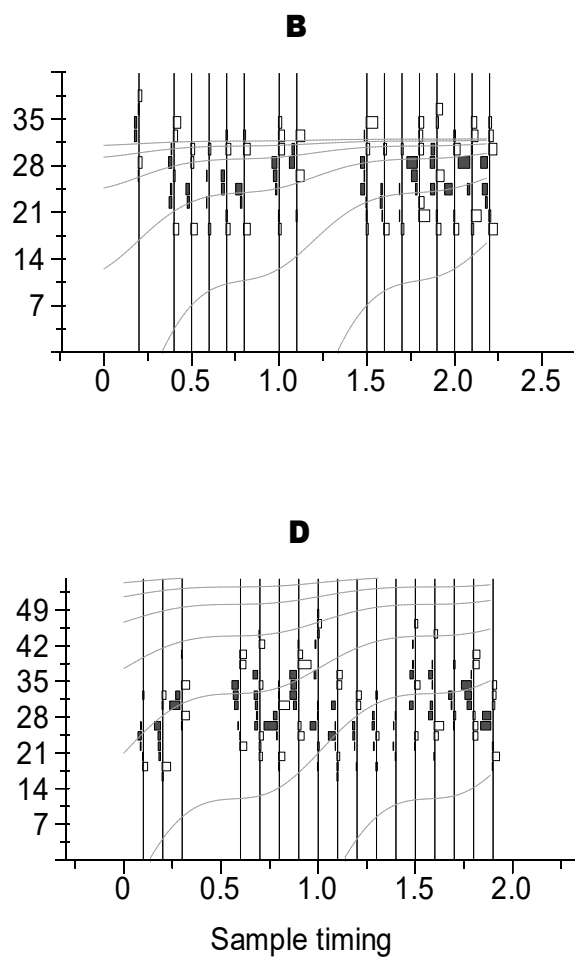

Fig. 3. Growth curves of Prochilodus nigricans caught in four Amazonian rivers: (A) Amazonas 1995-1996, (B) Japurá 1998-2000, (C) Madeira 1995-1996, and (D) Purus 1995-1996

A

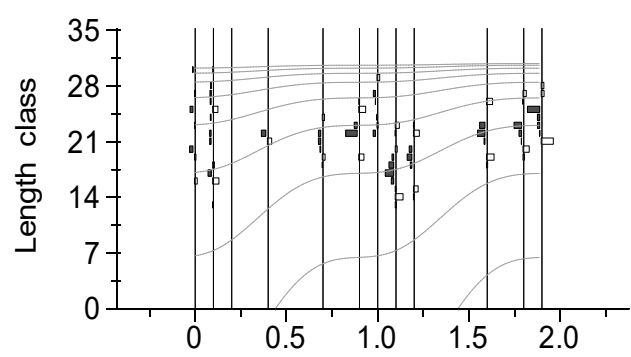

C

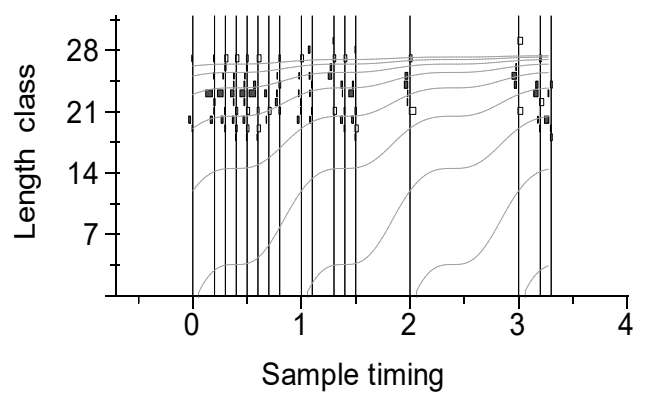

B

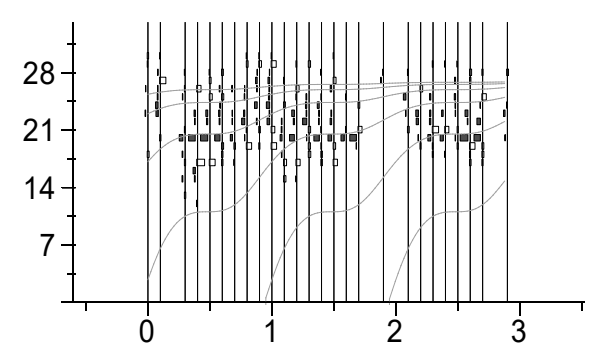

D

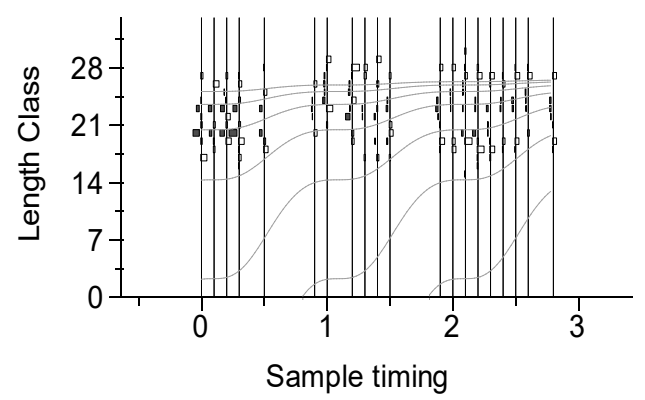

Fig. 4. Growth curves of Semaprochilodus insignis caught in two Amazonian rivers: Negro (A) 1995-1996, (B) 19982000, and (C) 2001-2004; and (D) Purus 2002-2004 
Cunha N.L., Catella A.C., Kinas M.A. 2007. Growth parameters estimates for small fish of the Pantanal, Brazil: Moenkhausia dichroura (Characiformes; Characidae). Brazilian Journal of Biology 67 (2): 293-7. DOI: 10.1590/S1519-69842007000200014

Escobar L.M.D., Andrade-López J., Farias I.P., Hrbek T. 2015. Delimiting evolutionarily significant units of the fish, Piaractus brachypomus (Characiformes: Serrasalmidae), from the Orinoco and Amazon river basins with insight on routes of historical connectivity. Journal of Heredity 106 (S1): 428-438. DOI: 10.1093/ jhered/esv047

Freitas C.E.C., Siqueira-Souza F.K., Humston R., Hurd L.E. 2013. An initial assessment of drought sensitivity in Amazonian fish communities. Hydrobiologia 705 (1): 159-171. DOI: 10.1007/s10750-012-1394-4

Froese R., Pauly D. (eds.) 2018. FishBase. [Version 02/2018] www.fishbase.org

García-Berthou E., Carmona-Catot G., Merciai R., Ogle D.H. 2012. A technical note on seasonal growth models. Reviews in Fish Biology and Fisheries 22 (3): 635-640. DOI: 10.1007/s11160-012-9262-x

Gomiero L.M., Carmassi A.L., Braga F.M.S. 2007. Crescimento e mortalidade de Brycon opalinus (Characiformes, Characidae) no Parque Estadual da Serra do Mar, Mata Atlântica, Estado de São Paulo. [Growth and mortality of Brycon opalinus (Characiforms, Characidae) in Serra do Mar State Park, Atlantic Rainforest, São Paulo State.] Biota Neotropica 7 (1): 21-26. [In Portuguese.] DOI: 10.1590/S1676-06032007000100002

Guerreiro A. 2017. Influência de extremos de cheia e de seca nos recursos capturados pela pesca comercial desembarcados no porto de Manaus (Amazonas, Brasil). [Influence of drought and flood extremes on commercial fishery resources, landed at the Manaus harbour (AM, Brazil).] Doctoral thesis, INPA, BADPI, Manaus, AM, Brazil. [In Portuguese.]

Hoenig N.A., Hanumara R.C. 1990. An empirical comparison of seasonal growth models. Fishbyte 8 (1): $32-34$.
A

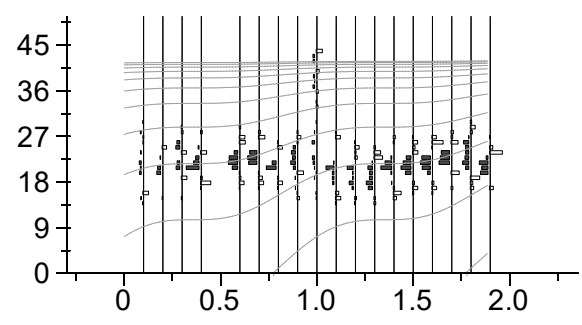

C

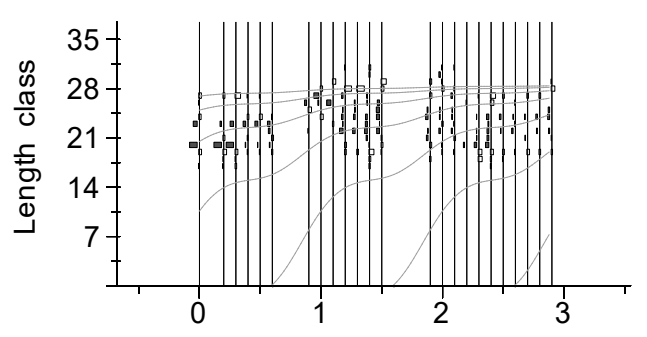

E

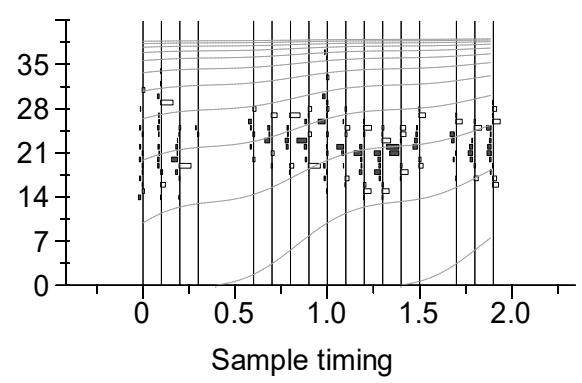

B

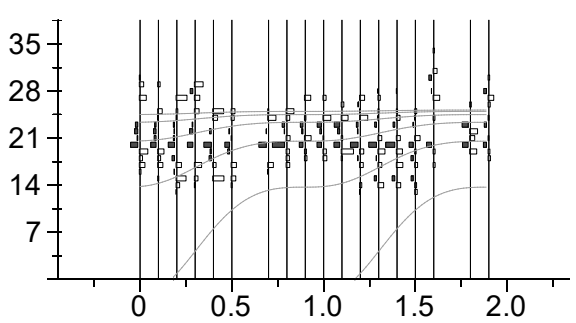

D

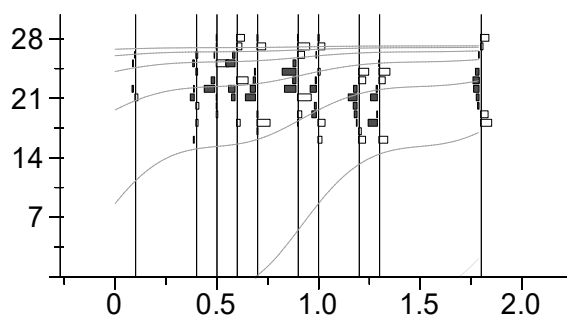

F

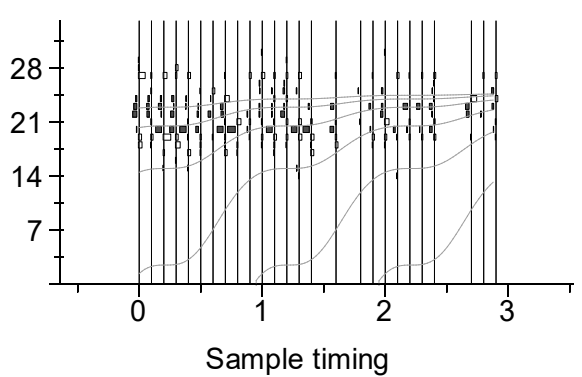

Fig. 5. Growth curves of Semaprochilodus insignis caught in three Amazonian rivers: Amazonas (A) 1995-1996, (B) 1999-2000, and (C) 2002-2004; (D) Juruá 1995-1996; and Madeira (E) 1995-1996, and (F) 1999-2001 

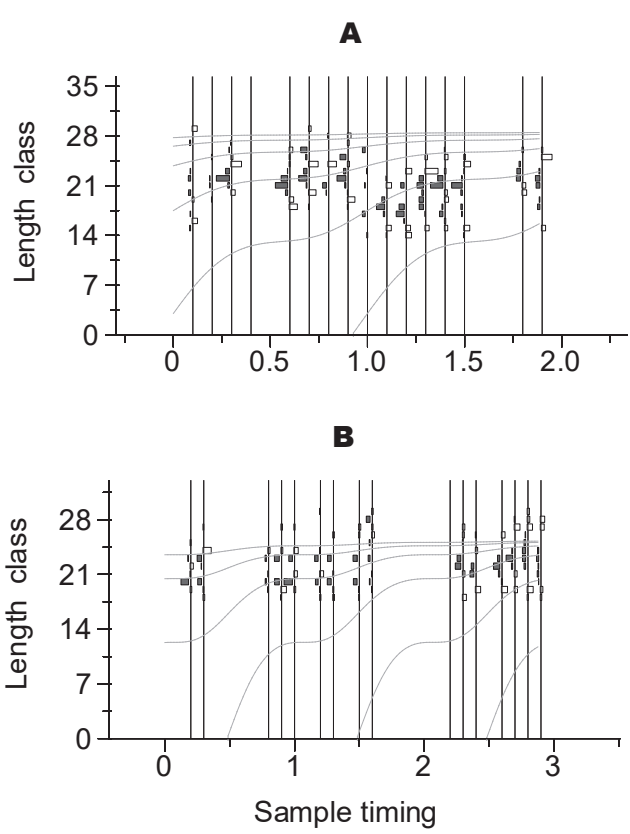

Fig. 6. Growth curves of Semaprochilodus taeniurus caught in the Amazonas river: (A) 1995-1996, and (B) 2001-2003
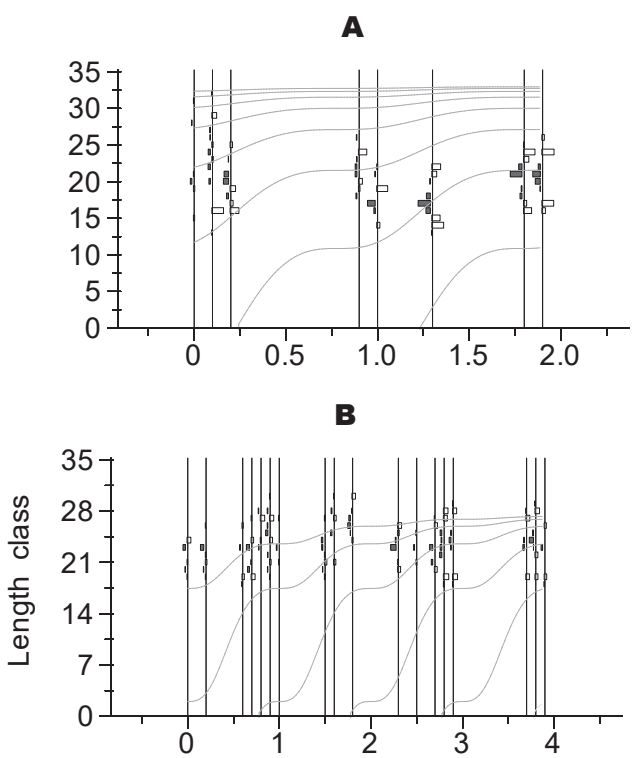

c

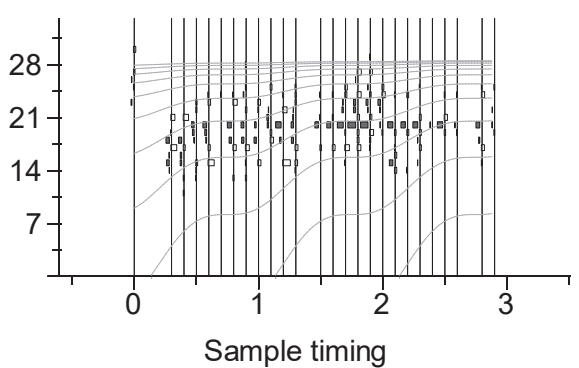

Fig. 7. Growth curves of Semaprochilodus taeniurus caught in three Amazonian rivers: (A) Madeira 1995-1996; (B) Negro 2001-2004; and (C) Purus 1998-2000
Isaac V.J., Almeida M.C., Giarrizo M., Deus C.P., Vale R., Klein G., Begossi A. 2015. Food consumption as an indicator of the conservation of natural resources in riverine communities of the Brazilian Amazon. Anais da Academia Brasileira de Ciências 87 (4): 2229-2242. DOI: $10.1590 / 0001-3765201520140250$

Isaac V.J., Fabré N.N., Silva C.O., Ruffino M.L., SaintPaul U. 2012. Ecologia da Fauna Ictíica. [Ecology of ichthyofauna.] Pp. 207-249. In: Batista V.S., Isaac V.J., Fabré N.N., Gonzalez J.C.G., de Almeida O.T., Rivero S., de Oliveira Júnior J.N., Ruffino M.L., Silva C.O. Saint-Paul U. (eds.) Peixes e pesca SolimõesAmazonas: uma avaliação integrada. [Fishes and fishery in Solimões-Amazonas: An integrated evaluation.] IBAMA/ProVárzea, Brasília, Brazil. [In Portuguese.]

Isaac V.J., Ruffino M.L. 1996. Population dynamics of tambaqui, Colossoma macropomum Cuvier, in the lower Amazon, Brazil. Fisheries Management and Ecology 3 (4): 315-333. DOI: 10.1046/j.13652400.1996.d01-154.x
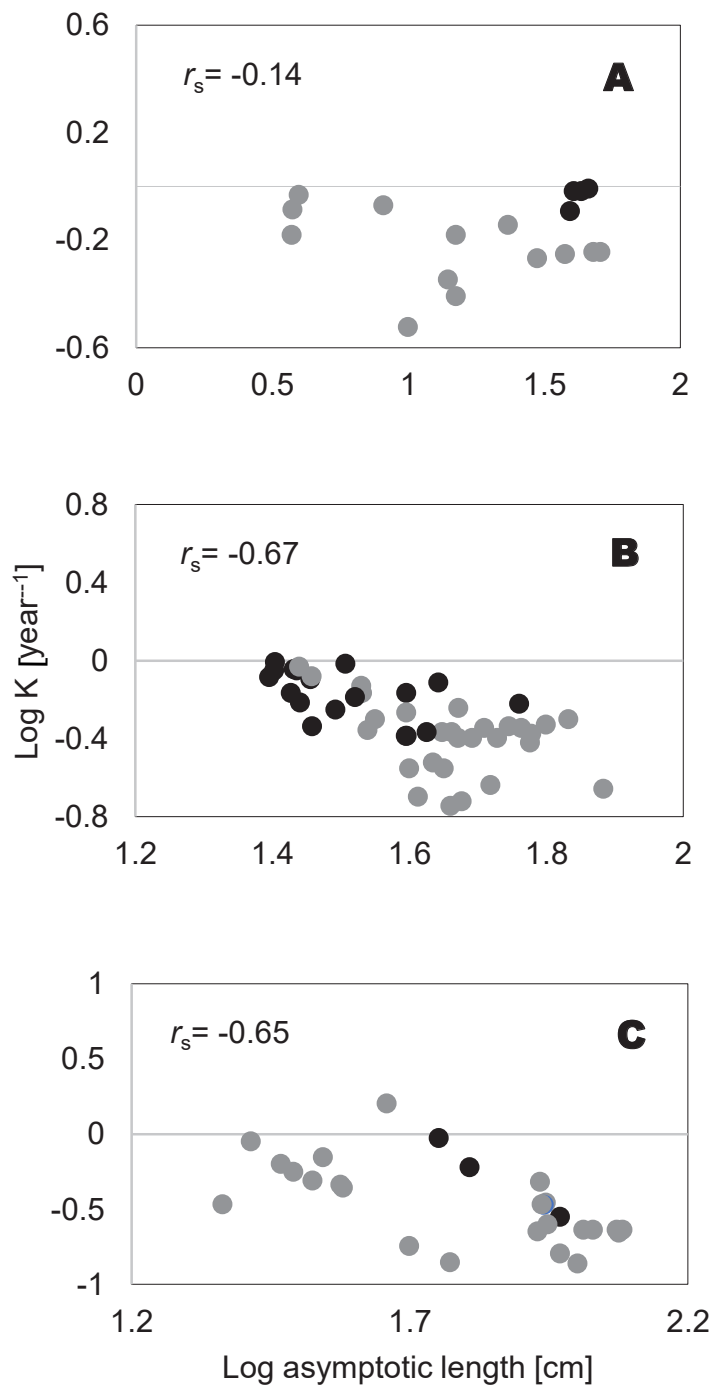

Fig. 8. Auximetric plots of (A) Characidae, (B) Prochilodontidae, and (C) Serrasalmidae; growth parameters: black circles, this study; grey circles, other studies 
Junk W.J. 1983. 4. Aquatic habitats in Amazonia. The Environmentalist 3 (Suppl. 5): 24-34. DOI: 10.1016/ S0251-1088(83)90199-7

King M. 2007. Fisheries biology, assessment and management. Blackwell Publishing, Oxford, UK.

Kirkwood G., Aukland R., Zara S. 2001. Length frequency distribution analysis (LFDA). MRAG Ltd., London, UK.

Li W., Zhang P., Ye J., Li L., Baker P.A. 2011. Impact of two different types of El Niño events on the Amazon climate and ecosystem productivity. Journal of Plant Ecology 4 (1-2): 91-99. DOI: 10.1093/jpe/rtq039

Lizama M.A.P., Ambrósio A.M. 2003. Crescimento, recrutamento e mortalidade do pequi Moenkhausia intermédia (Osteichthyes, Characidae) na planície de inundação do alto rio Paraná, Brasil. [Growth, recruitment and mortality of pequi Moenkhausia intermedia (Osteichthyes, Characidae) in the floodplains of the upper Paraná river, Brazil.] Acta
Scientiarum Biological Sciences 25 (2): 329-333. [In Portuguese.] DOI: 10.4025/actascibiolsci.v25i2.2020

Lourenço L.S., Fernandes I.M., Súarez Y.R. 2012. Spatial and temporal variation in the population structure of Hemigrammus marginatus (Characiformes: Characidae) in streams of the Ivinhema River basin, Brazil. Zoologia (Curitiba) 29 (4): 300-307. DOI: $10.1590 / \mathrm{S} 1984-46702012000400003$

Lopes G.C.S.L., Catarino M.F., Lima A.C., Freitas E.C. 2016. Small-scale fisheries in the Amazon basin: General patterns and diversity of fish landings in five sub-basins. Boletim do Instituto de Pesca 42 (4): 889-900. DOI: 10.20950/1678-2305.2016v42n4p889

Pardo S.A., Cooper A.B., Dulvy N.K. 2013. Avoiding fishy growth curves. Methods in Ecology and Evolution 4 (4): 353-360. DOI: 10.1111/2041-210x.12020

Pauly D. 1998. Tropical fishes: Patterns and propensities. Journal of Fish Biology 53 (Suppl. A): 1-17. DOI: 10.1111/j.1095-8649.1998.tb01014.x

Table 1

Complementary information, for the estimation of growth parameters of five species from six Amazonian rivers

\begin{tabular}{|c|c|c|c|c|c|}
\hline \multirow[b]{2}{*}{ Species } & \multicolumn{2}{|c|}{ First maturity } & \multirow[b]{2}{*}{ River } & \multirow[b]{2}{*}{ Year } & \multirow{2}{*}{$\begin{array}{l}\text { Maximum size } \\
\text { (FL) }[\mathrm{cm}]\end{array}$} \\
\hline & $\begin{array}{l}\text { Length } \\
(\mathrm{FL})[\mathrm{cm}]\end{array}$ & $\begin{array}{c}\text { Age } \\
{[\text { year] }}\end{array}$ & & & \\
\hline \multirow[t]{4}{*}{ Brycon amazonicus } & $37^{1}$ & $2^{2}$ & Amazonas & $2003-2004$ & 46 \\
\hline & & & Japurá & $1998-2000$ & 48 \\
\hline & & & Juruá & $1998-2000$ & 46 \\
\hline & & & Purus & 2003-2004 & 46 \\
\hline \multirow[t]{3}{*}{ Piaractus brachypomus } & $59^{3}$ & $3^{4}$ & Amazonas & $2000-2004$ & 68 \\
\hline & & & Japurá & 1998-2004 & 76 \\
\hline & & & Purus & 1999-2004 & 70 \\
\hline \multirow[t]{4}{*}{ Prochilodus nigricans } & $27^{1}$ & $2^{5}$ & Amazonas & 1995-1996 & 44 \\
\hline & & & Japurá & $1998-2000$ & 38 \\
\hline & & & Madeira & 1995-1996 & 48 \\
\hline & & & Purus & 1995-1996 & 50 \\
\hline \multirow[t]{10}{*}{ Semaprochilodus insignis } & $23^{1}$ & $2^{6}$ & Amazonas & 1995-1996 & 46 \\
\hline & & & & 1999-2000 & 35 \\
\hline & & & & $2002-2004$ & 34 \\
\hline & & & Juruá & 1995-1996 & 28 \\
\hline & & & Madeira & 1995-1996 & 38 \\
\hline & & & & 1999-2001 & 31 \\
\hline & & & Negro & 1995-1996 & 32 \\
\hline & & & & $1998-2000$ & 31 \\
\hline & & & & 2001-2004 & 29 \\
\hline & & & Purus & $2002-2004$ & 31 \\
\hline \multirow[t]{5}{*}{ Semaprochilodus taeniurus } & $22^{1}$ & $2^{6}$ & Amazonas & 1995-1996 & 32 \\
\hline & & & & $2001-2003$ & 30 \\
\hline & & & Madeira & 1995-1996 & 32 \\
\hline & & & Negro & 2001-2004 & 32 \\
\hline & & & Purus & $1998-2000$ & 31 \\
\hline
\end{tabular}

Values of the length at first maturity, available in the literature, were converted into fork length (FL) using the species-specific, lengthlength equation, which is available in Froese and Pauly (2018); ${ }^{1}$ Santos et al. 2006, ${ }^{2}$ Lopes et al. 2016, ${ }^{3}$ Froese and Pauly $2018,{ }^{4}$ Escobar et al. 2015, ${ }^{5}$ Santana and Freitas 2013, ${ }^{6}$ Vieira unpublished*.

"Vieira E. 2003. Dinâmica sazonal e interanual da estrutura populacional e do impacto da exploração pesqueira do Jaraqui de escama fina (Semaprochilodus taeniurus) e Jaraqui escama grossa (S. insignis) (Schomburgk, 1841) em subsistemas hidrográficos da Amazônia Central. [Seasonal and interannual dynamics of the population structure and the impact of fisheries exploitation of silver prochilodus (Semaprochilodus taeniurus) and kissing prochilodus (S. insignis) (Schomburgk, 1841) on hydrographic subsystems of Central Amazonia.] Doctoral thesis, INPA/UFAM, Manaus, Brazil. [In Portuguese.] 
Growth parameters of Characidae, Prochilodontidae, and Serrasalmidae

\begin{tabular}{|c|c|c|c|}
\hline Species & $L \infty$ & $K$ & Reference \\
\hline \multirow[t]{3}{*}{ Astyanax altiparanae Garutti et Britski, 2000} & 14.0 & 0.45 & Froese and Pauly 2018 \\
\hline & 15.0 & 0.39 & \\
\hline & 15.0 & 0.66 & \\
\hline \multirow[t]{6}{*}{ Brycon amazonicus (Spix et Agassiz, 1829) } & 43.3 & 0.96 & This study \\
\hline & 40.6 & 0.96 & \\
\hline & 39.4 & 0.81 & \\
\hline & 46.1 & 0.98 & \\
\hline & 51.0 & 0.57 & Santos Filho and Batista 2009 \\
\hline & 48.0 & 0.57 & Isaac et al. 2012 \\
\hline \multirow{2}{*}{ Brycon opalinus (Cuvier, 1819) } & 29.8 & 0.54 & Gomiero et al. 2007 \\
\hline & 37.7 & 0.56 & \\
\hline Hemigrammus marginatus Ellis, 1911 & 3.7 & 0.66 & Lourenço et al. 2012 \\
\hline Moenkhausia dichroura (Kner, 1858) & 8.1 & 0.85 & Cunha et al. 2007 \\
\hline Moenkhausia intermedia Eigenmann, 1908 & 10.0 & 0.30 & Lizama and Ambrósio 2003 \\
\hline Odontostilbe pequira (Steindachner, 1882) & 4.0 & 0.93 & Tondato et al. 2012 \\
\hline Oligosarcus hepsetus (Cuvier, 1829) & 23.3 & 0.72 & Carmassi et al. 2011 \\
\hline Prochilodus brevis Steindachner, 1875 & 47.0 & 0.57 & Froese and Pauly 2018 \\
\hline \multirow[t]{12}{*}{ Prochilodus lineatus (Valenciennes, 1837) } & 33.9 & 0.74 & Froese and Pauly 2018 \\
\hline & 34.0 & 0.68 & \\
\hline & 39.5 & 0.41 & \\
\hline & 44.5 & 0.43 & \\
\hline & 45.9 & 0.43 & \\
\hline & 47.5 & 0.19 & \\
\hline & 49.2 & 0.40 & \\
\hline & 51.2 & 0.45 & \\
\hline & 52.3 & 0.23 & \\
\hline & 53.5 & 0.40 & \\
\hline & 76.5 & 0.22 & \\
\hline & 55.7 & 0.46 & Vicentin et al. 2012 \\
\hline \multirow[t]{5}{*}{ Prochilodus magdalenae Steindachner, 1879} & 39.4 & 0.54 & Froese and Pauly 2018 \\
\hline & 43.1 & 0.30 & \\
\hline & 44.7 & 0.28 & \\
\hline & 59.8 & 0.38 & \\
\hline & 60.0 & 0.42 & \\
\hline Prochilodus mariae Eigenmann, 1922 & 46.9 & 0.40 & Pérez-Lozano and Aniello 2013 \\
\hline \multirow[t]{10}{*}{ Prochilodus nigricans Spix et Agassiz, 1829} & 39.5 & 0.68 & This study \\
\hline & 32.1 & 0.96 & \\
\hline & 43.9 & 0.77 & \\
\hline & 57.7 & 0.60 & \\
\hline & 68.0 & 0.50 & Ruffino and Isaac 1995 \\
\hline & 58.0 & 0.45 & \\
\hline & 34.6 & 0.44 & Catarino et al. 2014 \\
\hline & 45.8 & 0.18 & Silva and Stewart 2006 \\
\hline & 39.8 & 0.28 & \\
\hline & 63.0 & 0.47 & Isaac et al. 2012 \\
\hline Prochilodus reticulatus Valenciennes, 1850 & 41.0 & 0.20 & Froese and Pauly 2018 \\
\hline \multirow[t]{7}{*}{ Semaprochilodus insignis (Jardine, 1841) } & 42.2 & 0.43 & This study \\
\hline & 25.3 & 0.89 & \\
\hline & 28.6 & 0.80 & \\
\hline & 27.3 & 0.89 & \\
\hline & 39.4 & 0.41 & \\
\hline & 24.9 & 0.82 & \\
\hline & 31.1 & 0.56 & \\
\hline
\end{tabular}


Table 2 cont.

\begin{tabular}{|c|c|c|c|}
\hline Species & $L \infty$ & $K$ & Reference \\
\hline & 27.0 & 0.90 & \\
\hline & 27.6 & 0.61 & \\
\hline & 26.8 & 0.68 & \\
\hline \multirow[t]{6}{*}{ Semaprochilodus taeniurus (Valenciennes, 1821) } & 28.7 & 0.83 & This study \\
\hline & 25.4 & 0.98 & \\
\hline & 33.2 & 0.65 & \\
\hline & 27.5 & 0.93 & \\
\hline & 28.7 & 0.46 & \\
\hline & 35.5 & 0.50 & Isaac et al. 2012 \\
\hline \multirow[t]{9}{*}{ Colossoma macropomum (Cuvier, 1816) } & 121.0 & 0.23 & Ruffino and Isaac 1995 \\
\hline & 118.0 & 0.23 & \\
\hline & 119.9 & 0.23 & Isaac and Ruffino 1996 \\
\hline & 107.0 & 0.23 & Froese and Pauly 2018 \\
\hline & 88.7 & 0.25 & Pérez-Lozano and Aniello 2013 \\
\hline & 119.0 & 0.22 & Isaac et al. 2012 \\
\hline & 85.1 & 0.23 & Penna et al. 2005 \\
\hline & 100.4 & 0.14 & \\
\hline & 93.3 & 0.16 & Villacorta-Corrêa unpublished ${ }^{*}$ \\
\hline Myloplus rhomboidalis (Cuvier, 1818) & 38.0 & 0.44 & Froese and Pauly 2018 \\
\hline \multirow[t]{2}{*}{ Mylossoma duriventre (Cuvier, 1818) } & 33.5 & 0.49 & Pérez-Lozano and Aniello 2013 \\
\hline & 31.0 & 0.56 & Isaac et al. 2012 \\
\hline \multirow[t]{6}{*}{ Piaractus brachypomus (Cuvier, 1818) } & 56.6 & 0.94 & This study \\
\hline & 93.4 & 0.28 & \\
\hline & 64.3 & 0.60 & \\
\hline & 45.6 & 1.59 & Froese and Pauly 2018 \\
\hline & 102.9 & 0.23 & Pérez-Lozano and Aniello 2013 \\
\hline & 88.0 & 0.35 & Isaac et al. 2012 \\
\hline \multirow[t]{5}{*}{ Piaractus mesopotamicus (Holmberg, 1887) } & 50.0 & 0.18 & Ambrósio et al. 2014 \\
\hline & 59.2 & 0.14 & \\
\hline & 87.2 & 0.34 & Peixer et al. 2007 \\
\hline & 86.5 & 0.34 & \\
\hline & 86.0 & 0.48 & Vaz unpublished ${ }^{*}$ \\
\hline Pygocentrus cariba (von Humboldt, 1821) & 37.6 & 0.46 & Pérez-Lozano and Aniello 2013 \\
\hline \multirow[t]{3}{*}{ Pygocentrus nattereri Kner, 1858} & 26.0 & 0.89 & Froese and Pauly 2018 \\
\hline & 29.4 & 0.63 & Bevilaqua and Soares 2010 \\
\hline & 35.0 & 0.70 & Isaac et al. 2012 \\
\hline Serrasalmus spilopleura Kner, 1858 & 23.1 & 0.34 & Sousa et al. 2013 \\
\hline
\end{tabular}

$L \infty=$ asymptotic length, $K=$ growth coefficient. 
Table 3

Growth parameters estimates for five species of six Amazonian rivers

\begin{tabular}{|c|c|c|c|c|c|c|c|c|}
\hline \multirow{2}{*}{ Species } & \multirow{2}{*}{ River } & \multirow{2}{*}{ Year } & \multirow{2}{*}{$N$} & \multicolumn{5}{|c|}{ Results } \\
\hline & & & & $L_{\infty}$ & $K$ & $t_{0}$ & $C$ & WP \\
\hline \multirow[t]{4}{*}{ Brycon amazonicus } & Amazonas & $2003-2004$ & 911 & 43.29 & 0.96 & -0.39 & 0.99 & Dec \\
\hline & Japurá & $1998-2000$ & 819 & 40.63 & 0.96 & -0.64 & 1.00 & Dec \\
\hline & Juruá & $1998-2000$ & 1942 & 39.38 & 0.81 & -0.94 & 0.99 & Apr \\
\hline & Purus & $2003-2004$ & 736 & 46.07 & 0.98 & -0.23 & 0.93 & Jan \\
\hline \multirow[t]{3}{*}{ Piaractus brachypomus } & Amazonas & $2000-2004$ & 591 & 56.57 & 0.94 & -0.14 & 1.00 & May \\
\hline & Japurá & $1998-2004$ & 1147 & 93.43 & 0.28 & -0.83 & 1.00 & Oct \\
\hline & Purus & 1999-2004 & 1468 & 64.29 & 0.60 & -0.61 & 1.00 & Sep \\
\hline \multirow[t]{4}{*}{ Prochilodus nigricans } & Amazonas & 1995-1996 & 1643 & 39.45 & 0.68 & -0.94 & 0.76 & Sep \\
\hline & Japurá & $1998-2000$ & 830 & 32.14 & 0.96 & -0.71 & 0.99 & Oct \\
\hline & Madeira & $1995-1996$ & 696 & 43.90 & 0.77 & -0.40 & 0.31 & Nov \\
\hline & Purus & 1995-1996 & 1673 & 57.65 & 0.60 & -0.87 & 0.96 & Jul \\
\hline \multirow[t]{10}{*}{ Semaprochilodus insignis } & \multirow{3}{*}{ Amazonas } & 1995-1996 & 2920 & 42.21 & 0.43 & -0.24 & 1.00 & May \\
\hline & & $1999-2000$ & 6667 & 25.29 & 0.89 & -0.83 & 1.00 & Nov \\
\hline & & 2002-2004 & 4216 & 28.57 & 0.80 & -0.38 & 0.83 & May \\
\hline & Juruá & 1995-1996 & 423 & 27.29 & 0.89 & -0.30 & 0.83 & Mar \\
\hline & \multirow{2}{*}{ Madeira } & 1995-1996 & 1331 & 39.39 & 0.41 & -0.64 & 0.75 & May \\
\hline & & 1999-2001 & 3449 & 24.86 & 0.82 & -0.08 & 1.00 & Mar \\
\hline & \multirow{3}{*}{ Negro } & 1995-1996 & 544 & 31.09 & 0.56 & -0.56 & 0.94 & Nov \\
\hline & & $1998-2000$ & 7109 & 27.00 & 0.90 & -0.05 & 1.00 & Jun \\
\hline & & 2001-2004 & 1241 & 27.57 & 0.61 & -0.90 & 1.00 & May \\
\hline & Purus & $2002-2004$ & 2927 & 26.75 & 0.68 & -0.20 & 1.00 & Feb \\
\hline \multirow[t]{5}{*}{ Semaprochilodus taeniurus } & \multirow{2}{*}{ Amazonas } & $1995-1996$ & 1122 & 28.69 & 0.83 & -0.08 & 0.82 & July \\
\hline & & $2001-2003$ & 1850 & 25.36 & 0.98 & -0.53 & 1.00 & Jan \\
\hline & Madeira & 1995-1996 & 563 & 33.17 & 0.65 & -0.77 & 1.00 & Oct \\
\hline & Negro & $2001-2004$ & 1310 & 27.50 & 0.93 & -0.23 & 1.00 & Dec \\
\hline & Purus & 1998-2000 & 4346 & 28.73 & 0.46 & -0.87 & 1.00 & Oct \\
\hline
\end{tabular}

$L_{\infty}=$ asymptotic length, $K=$ growth coefficient, $t_{0}=$ theoretical age at zero length, $C=$ relative amplitude of seasonal oscillations, WP $=$ winter point.

Pauly D., David N. 1981. ELEFAN I, a BASIC program for the objective extraction of growth parameters from length-frequency data. Berichte der Deutschen wissenschaftlichen Kommission für Meeresforschung 28 (4): 205-211.

Peixer J., Catella A.C., Petrere Júnior M. 2007. Yield per recruit of the pacu Piaractus mesopotamicus (Holmberg, 1887) in the pantanal of Mato Grosso do Sul, Brazil. Brazilian Journal of Biology 67 (3): 561-567. DOI: 10.1590/S1519-69842007000300023

Penna M.A.H., Villacorta-Corrêa M.A., Walter T., Petrere-Jr M. 2005. Growth of tambaqui Colossoma macropomum (Cuvier) (Characiformes: Characidae): Which is the best model? Brazilian Journal of Biology 65 (1):129-139. DOI: 10.1590/S151969842005000100017
Pérez-Lozano A., Aniello B. 2013. Parámetros poblacionales de los principales recursos pesqueros de la cuenca del río Apure, Venezuela (2000-2003). Latin American Journal of Aquatic Research 41 (3): 447-458. DOI: 10.3856/vol41-issue3-fulltext-8

Ruffino M.L., Isaac V.J. 1995. Life cycle and biological parameters of several Brazilian Amazon fish species. Naga, the ICLARM Quarterly 18 (4): 41-45.

Santana I.F., Freitas C.E.C. 2013. A time series analysis of Prochilodus nigricans landings caught by smallscale fisheries in the lower stretch of the Amazon River. Brazilian Journal of Biology 73 (1): 53-59. DOI: $10.1590 / \mathrm{S} 1519-69842013000100007$

Santos G., Ferreira E., Zuanon J. 2006. Peixes comerciais de Manaus. [Commercial fishes of Manaus.] IBAMA, PROVÁRZEA, Manaus, Brazil. [In Portuguese.] 
Santos FilhoL.C.,BatistaV.S.2009.Dinâmicapopulacional da matrinxã Brycon amazonicus (Characidae) na Amazônia central. [Population dynamics of matrinxã, Brycon amazonicus (Characidae) in central Amazon. Zoologia (Curitiba) 26: 195-203. [In Portuguese.] DOI: 10.1590/S1984-46702009000200001

Silva E.A., Stewart D.J. 2006. Age structure and survival rates of the commercial fish Prochilodus nigricans (bocachico) in north-eastern Ecuador. Environmental Biology of Fishes 77 (1): 63-77. DOI: 10.1007/ s10641-006-9055-y

Sousa F.B., Soares M.G.M., Prestes L. 2013. Population dynamics of the yellow piranha Serrasalmus spilopleura Kner, 1858 (Characidae, Serrasalmidae) in the Amazonian floodplain lakes. Acta Scientiarum Biological Sciences 35 (3): 367-372. DOI: 10.4025/ actascibiolsci.v35i3.15749

Tomasella J., Pinho P.F., Borma L.S., Marengo J.A., Nobre C.A., Bittencourt O.R.F.O., Prado M.C.R., Rodriguez D.A., Cuartas L.A. 2012. The droughts of 1997 and 2005 in Amazonia: Floodplain hydrology and its potential ecological and human impacts. Climatic Change 116 (3-4): 723-746. DOI: 10.1007/ s10584-012-0508-3

Tondato K.K., Fialho C.B., Súarez Y.R. 2012. Life history traits of Odontostilbe pequira (Steindachner, 1882 ) in the pantanal of Porto Murtinho, Mato Grosso do Sul State, Brazil. Oecologia Australis 16 (4): 878-890. DOI: 10.4257/oeco.2012.1604.11

Vicentin W., Rocha A.S., Rondon P.L, Costa E.S.C., Súarez Y. 2012. Parâmetros populacionais, período reprodutivo e crescimento de Prochilodus lineatus (Characiformes, Prochilodontidae) na cabeceira do rio Miranda, alto rio Paraguai. [Populational parameters, reproductive period and growth of Prochilodus lineatus (Characiformes, Prochilodontidae) in the headwaters of Miranda River, Upper Paraguai River. Oecologia Australis 16 (4): 891-904. [In Portuguese.] DOI: $10.4257 /$ oeco.2012.1604.12

Received: 20 November 2017 Accepted: 24 May 2018 Published electronically: 30 September 2018 